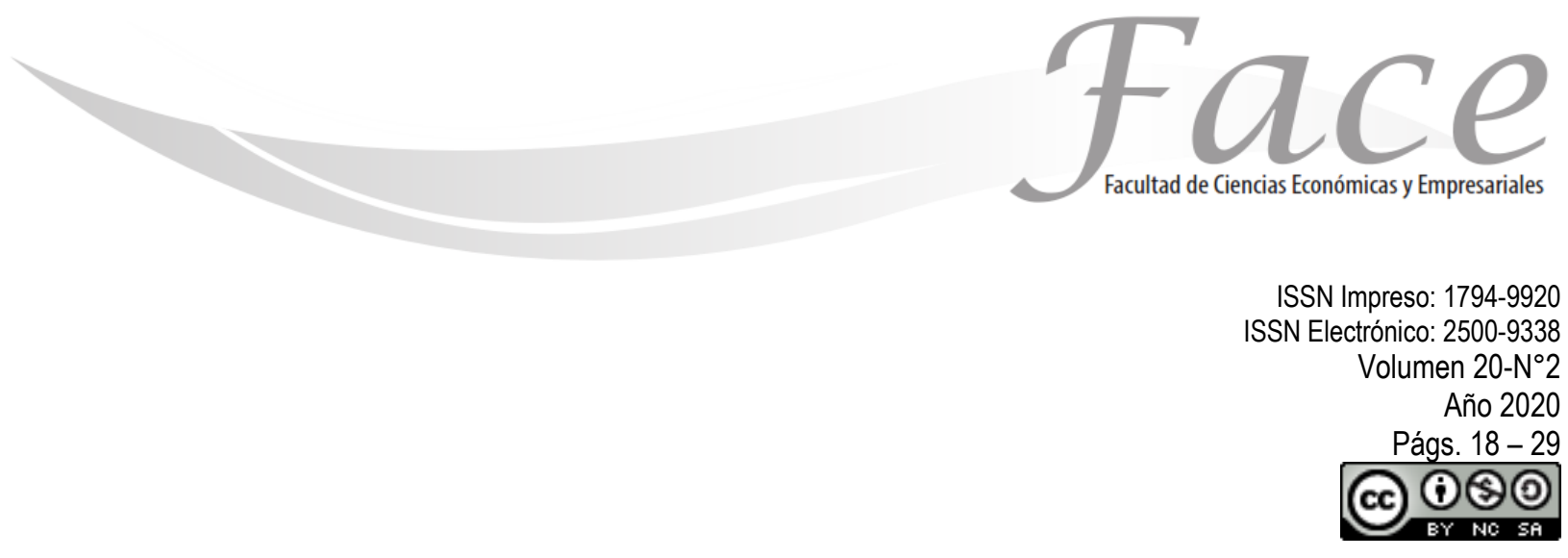

\title{
RELACIÓN DE LA ORIENTACIÓN AL CLIENTE, DE VALOR AL CLIENTE Y LA INNOVACIÓN SOBRE EL DESEMPEÑO ORGANIZACIONAL. UN ESTUDIO EN MIPYMES DEL ESTADO DE HIDALGO, MÉXICO
}

\author{
Juan Gabriel Figueroa Velázquez* \\ Enlace ORCID: https://orcid.org/0000-0002-8846-1359 \\ María de Monserrat López Moreno** \\ Enlace ORCID: https://orcid.org/0000-0002-3429-9659 \\ Dra. Alejandra Vega Barrios ** \\ Enlace ORCID: https://orcid.org/0000-0002-2368-7939
}

Fecha de Recepción: Septiembre 17 de 2020

Fecha de Aprobación: Diciembre 19 de 2020

\section{Resumen:}

El objetivo de la presente investigación fue validar la relación entre la orientación al cliente, las acciones de innovación, el valor al cliente y el desempeño organizacional en mipymes establecidas en el estado de Hidalgo, México, mediante la utilización del análisis de correlación. Utilizando el software Stata, se confirmó la relación positiva y significativa de la orientación al cliente y el valor al cliente con el desempeño organizacional. No se encontró apoyo que validara la relación entre la innovación y el desempeño. Estos resultados aportan evidencia empírica de la relación de estas variables en un país en desarrollo.

Palabras clave: orientación al cliente; innovación; desempeño organizacional, valor al cliente, pymes.

\footnotetext{
* Doctor en Planeación Estratégica y Dirección de Tecnología. Profesor Investigadora del Instituto de Ciencias Económico Administrativas, de la Universidad Autónoma del Estado de Hidalgo, México. Contacto: figueroa@uaeh.edu.mx

* * Estudiante de la Licenciatura en Mercadotecnia. Instituto de Ciencias Económico Administrativas, de la Universidad Autónoma del Estado de Hidalgo, México. Contacto: monserrattlm@gmail.com

*** Doctora en Proyectos con línea en Gestión Empresarial en la Universidad Internacional Iberoamericana. Profesor Investigadora del Instituto de Ciencias Económico Administrativas, de la Universidad Autónoma del Estado de Hidalgo, México. Contacto: vbarrios@uaeh.edu.mx
} 


\title{
RELATIONSHIP OF CUSTOMER ORIENTATION, CUSTOMER VALUE AND INNOVATION ON ORGANIZATIONAL PERFORMANCE. A STUDY IN MSMES IN THE STATE OF HIDALGO, MEXICO.
}

\begin{abstract}
:
The objective of this research was to validate the relationship between customer orientation, innovation actions, customer value and organizational performance in MSMEs established in the state of Hidalgo, Mexico, through the use of correlation analysis. Using Stata software, the positive and significant relationship of customer orientation and customer value with organizational performance was confirmed. No support was found to validate the relationship between innovation and performance. These results provide empirical evidence of the relationship of these variables in a developing country.
\end{abstract}

Keywords Customer orientation; innovation; organizational performance, customer value, SMEs.

\section{RELACIONAMENTO DE ORIENTAÇÃO PARA O CLIENTE, VALOR PARA O CLIENTE E INOVAÇÃO NO DESEMPENHO ORGANIZACIONAL. UM ESTUDO EM MIPIMOS NO ESTADO DE HIDALGO, MÉXICO}

Resumo:

O objetivo desta pesquisa foi validar a relação entre orientação para o cliente, ações de inovação, valor para o cliente e desempenho organizacional em MPMEs estabelecidas no estado de Hidalgo, México, por meio do uso de análise de correlação. Usando o software Stata, foi confirmada a relação positiva e significativa da orientação para o cliente e do valor para o cliente com o desempenho organizacional. Nenhum suporte foi encontrado para validar a relação entre inovação e desempenho. Esses resultados fornecem evidências empíricas da relação dessas variáveis em um país em desenvolvimento.

Palavras-chave: Orientação para o cliente; inovação; desempenho organizacional, valor para o cliente, PMEs. 


\section{INTRODUCCIÓN:}

\section{Antecedentes}

Actualmente las organizaciones necesitan dirigir sus esfuerzos a desarrollar actividades basadas en la orientación al cliente, si es que desean realmente lograr el éxito de sus negocios. Una de las causas de la competitividad que se vive en estos días es la globalización, lo que lleva a las empresas a estudiar constantemente a sus clientes, competidores y proveedores para obtener tanta información como sea posible y sobre esto tomar decisiones para proponer soluciones innovadoras que les permita continuar en el mercado (Valenzuela \& Villegas, 2015). Es por ello que, si realmente las organizaciones se concientizan acerca de la importancia que tiene colocar a los clientes como su objetivo principal, será porque conocen la capacidad que les genera esta orientación de colocarse en una posición estratégica frente a sus competidores (Acevedo et al., 2007), lo que significa, que la orientación al cliente tiene una relación directa con el desempeño organizacional.

En tal sentido, en la literatura revisada, se encontraron diversas investigaciones que muestran la relación positiva que existe entre la orientación al cliente y desempeño organizacional. Una de ellas realizada en Chile, concluyó que la orientación al cliente está vinculada positivamente con el desempeño organizacional (Valenzuela \& Martínez, 2015). Por tanto, la gestión con orientación a generar valor para el cliente realmente permite que una empresa se distinga sobre las demás, a través de un manejo eficiente de los recursos de mercadotecnia dirigidos a sus clientes más rentables.

Ahora bien, respecto a la variable valor al cliente, en la literatura revisada se encontraron muy pocas investigaciones que relacionen este constructo con el desempeño organizacional. Inicialmente puede comentarse que, de acuerdo con Porter (1991) las empresas deben buscar sobresalir de entre sus competidores a través de la generación de una ventaja competitiva, que les permita un mejor desempeño, para poder desarrollarla es necesario generar valor para el cliente a través del conocimiento de sus necesidades. Así mismo, las empresas que pretenden no sólo sobrevivir en el mercado, sino llegar al éxito, necesitan entregar valor a los clientes a través del conocimiento de los mismos, así como también de la competencia y su entorno, además de generar buenas relaciones con sus colaboradores, proveedores y distribuidores (Hernández, 2012).

Finalmente, acerca de la relación existente entre las variables innovación y desempeño organizacional, Yamakawa \& Ostos (2011) desarrollaron una investigación en Perú donde confirman la relación que existe entre estas dos variables, lo cual indica que las organizaciones llevan a cabo cambios en sus procesos, servicios y/o productos para lograr los objetivos organizacionales de desempeño. Para que una empresa pueda ser competitiva necesita generar cambios y nuevas creaciones, lo que en otras palabras quiere decir adoptar la innovación. Recomiendan que las organizaciones deben implementar innovaciones no sólo en los procesos productivos o técnicos, sino también en los procesos administrativos para lograr mejores niveles de desempeño.

-Planteamiento del problema

La globalización es una de las causas principales por las que se genera la competitividad en los mercados, si las empresas buscan realmente sobrevivir y permanecer deben desarrollar estudios constantes sobre sus clientes, competencia, proveedores para generar información y llevar a cabo el proceso de toma de decisiones correctamente (Valenzuela y Villegas, 2015).

En la revisión de la literatura realizada, se encontraron diversas publicaciones que relacionan la orientación al cliente con el desempeño organizacional, sin embargo Rodríguez et al., (2015), quienes estudiaron la relación entre la orientación al cliente y la orientación a la competencia en el desempeño organizacional de empresas mexicanas, sugieren como futuras investigaciones, el estudio más detallado de la relación entre estos constructos, así como analizar esta relación en otras regiones de México.

De igual forma, en Chile se desarrolló un estudio por Valenzuela y Villegas (2015), aplicado a distintas empresas de servicios, donde se midió el grado en que la orientación al valor del cliente por parte de la fuerza de ventas influye en el desempeño organizacional. Estos autores sugieren que estudios similares sean realizados en otros países de Latinoamérica, que estén interesados en una mejor gestión organizacional con orientación al mercado. 
En otros países también se han hecho investigaciones sobre la innovación y el desempeño organizacional. En Pakistán, Arshad et al., (2016) desarrollaron un estudio en el sector financiero que les permitió evaluar la relación entre la gestión total de calidad, la innovación de los servicios y el desempeño organizacional. El análisis de los resultados permitió concluir que la implementación de la gestión total de calidad tiene una relación positiva e importante con la innovación del servicio; sin embargo, se encontró una relación positiva pero débil entre la innovación del servicio y el desempeño organizacional. Esto da oportunidad a futuras investigaciones de poder afirmar la relación directa entre la innovación y el desempeño organizacional en países en desarrollo como México.

García et al., (2016) sugieren que es importante orientar las investigaciones sobre una innovación integral que incluya productos, procesos y sistemas de gestión y no sólo de productos, además de la relación de estas innovaciones con el desempeño organizacional y el impacto que genera.

En tal sentido el objetivo de esta investigación es validar la relación entre la orientación al cliente, las acciones de innovación, el valor al cliente y el desempeño organizacional experimentado en las mipymes establecidas en el estado de Hidalgo, México.

\section{MARCO TEORICO:}

\section{Desempeño organizacional}

La preocupación por documentar como las organizaciones

buscan alcanzar los objetivos y cumplir las metas que se establecen para una mejor eficacia y eficiencia, se remonta a los primeros estudios realizado por Taylor en 1914, más recientemente se formalizó la investigación de este constructo, mediante el trabajo desarrollado por Anand y Daft en el año 2007, lo que finalmente evolucionó y se terminó conceptualizando como desempeño organizacional (Machorro et al., 2015). En este sentido, el desempeño de una empresa debe darse por ciertas competencias, aptitudes, habilidades, motivaciones, percepciones de cada rol que se lleva a cabo en la organización, influidas por factores propios del empleado y del entorno (Churchill et al., 1985).

Gutiérrez et al., (2013) sugieren que una empresa requiere de capacitar a sus colaboradores en sus funciones de acuerdo a los requerimientos de la organización para lograr los niveles de desempeño que se buscan. Señalan además, que se deben mejorar los conocimientos, habilidades, actitudes y conductas de éstos colaboradores. Para Ogunyomi y Bruning (2015), el constructo desempeño organizacional debe agrupar variables como la ventaja competitiva, la participación de la empresa en el mercado, la satisfación del cliente e indicadores financieros como los ingresos por venta, los costos y la ganancia.

A través de diferentes investigaciónes se ha comprobado la relación de diferentes prácticas organizacionales con el desempeño de la empresa, como lo es el aprendizaje, la competitividad, la gestión de recursos humanos, la orientación al mercado, la innovación, etcétera (Almanza et al., 2016; Ynzunza y Izar, 2016; Valenzuela y Martínez, 2015; Yamakawa y Ostos, 2011). El desempeño organizacional se ha visto representado por indicadores como la productividad, la rentabilidad, las ventas, la satisfacción de los clientes, así como la lealtad de los mismos colaboradores (Ynzunza \& Izar, 2016).

\section{Orientación al cliente}

De acuerdo a la literatura revisada, coinciden distintos autores como González \& González (2004) o Ynzunza \& Izar (2013), que se considera a los trabajos desarrollados por Narver y Slater (1990) y Kohli y Jaworoski (1990) como los seminales relacionados con el constructor de orientación al cliente. Algunos académicos de la mercadotecnia como Llonch (1993) y Nwankwo (1995) sugieren que no hay una diferencia relevante de la orientación al cliente con la orientación al mercado, y es posible usar ambos términos para referir lo mismo. Aunque algunos autores dicen que la orientación al cliente es una dimensión de la orientación al mercado, la orientación al cliente debe ser prioritaria de entre las demás dimensiones, ya que conlleva un beneficio más grande para las empresas (Kohli y Jaworoski, 1990).

Narver y Slater (1990) sugieren que se debe ver a la orientación al mercado como una cultura organizacional que se compone por tres factores, los 
cuales son la orientación cliente, la orientación a la competencia y la coordinación de las funciones. La orientación al cliente y la orientación al mercado refieren a la obtención de datos y su difusión por toda la empresa, la coordinación de las funciones se refiere a la obtención de datos sobre el mercado para la creación y ofrecimiento de valor al cliente, mayor que la competencia. Consideran que la orientación al mercado permite desarrollar formas eficientes y eficaces para la creación de mayor valor frente a la competencia, esto debe estar basado en criterios de decisión, los cuales son dos: la rentabilidad y el enfoque a largo plazo.

Por otra parte, Kohli y Jaworoski (1990), definen a la orientación al mercado como el desarrollo o evolución del término mercadotecnia, el cual se basa en tres acciones: la creación de una inteligencia de mercado con información sobre las necesidades actuales y futuras del cliente, la difusión de esta inteligencia en cada área de la empresa y la forma en que la empresa puede generar una respuesta con esto. De acuerdo con Kohli y Jaworoski (1990), dependiendo de qué tan coordinados se encuentren las diferentes áreas funcionales de una empresa, será su orientación al mercado, desde la comunicación directa existente, pues esto genera una conexión que permite que el proceso de difusión de la inteligencia de mercadotecnia sea más fácil y eficiente, así como también facilita la respuesta que la empresa puede generar.

\section{Relación empírica entre la orientación al cliente y el desempeño organizacional}

En los últimos años se han desarrollado distintas investigaciones que estudian la relación de la orientación al cliente con distintas variables como el desempeño organizacional. Rodríguez et al. (2015) realizaron una investigación en micro, pequeñas y medianas empresas (Mipymes) mexicanas, quienes evaluaron las dimensiones de la orientación al mercado que proponen Narver y Slater (1990). Los resultados demostraron que existe una relación positiva y significativa entre el desempeño organizacional y la orientación al mercado. De las tres dimensiones, las que tuvieron una mayor correlación fueron la orientación al cliente y la orientación a la competencia.

Otra investigación realizada a una empresa líder del sector de consumo masivo en Chile (Valenzuela \& Martínez, 2015) concluyó que existe una relación positiva pero moderada de la orientación al cliente con el desempeño organizacional, ya que la gestión de las empresas con esta orientación les permite un mejor uso de los recursos de mercadotecnia y ventas enfocados en los clientes más rentables, generando mayores ingresos.

Es por ello que, debido a lo anteriormente expuesto, se propone la siguiente hipótesis:

H1: Las acciones de orientación al cliente que desarrollan las mipymes del estado de Hidalgo, México, influyen en su nivel de desempeño organizacional.

\section{La Innovación}

La innovación suele ser adoptada por las empresas como reacción a los cambios que pueden surgir en la gerencia y directivos, en los accionistas, en el ámbito tecnológico o en la competencia, todo con el propósito de mejorar el desempeño organizacional (Afcha, 2012). Las empresas implementan la innovación para poder lograr una ventaja competitiva a través de respuestas anticipadas, su valor radica en que hace a las organizaciones competitivas (Tushman y Nadler, 1986).

Puede considerarse a Damanpour (1996) como uno de los investigadores seminales en el constructo de la innovación (Yamakawa et al, 2011; Afcha, 2012; Toro et al., 2017). Para él, innovar es un procedimiento donde se genera, desarrolla e implementan ideas 0 comportamientos nuevos, la innovación surge como una respuesta preventiva y correctiva hacia los cambios constantes del entorno (Damanpour, 1996). Sugiere que existen diferentes tipos de innovación (administrativa y técnica) y guardan relación con las características de cada empresa, se vuelven asociaciones significativas como lo es la innovación y el desempeño organizacional. Para lograr que el grado de desempeño de una organización se mantenga y además mejore es necesaria la adopción de innovación tanto administrativa como técnica (Damanpour et al., 1989).

Ahora bien, existen diversas conceptualizaciones sobre la innovación. Seaden et. al. (2003) nos dicen que innovar es poner en marcha nuevos procesos, productos o enfoques de gestión para tener una mejor eficiencia y efectividad en las organizaciones. Para Damanpour (1996) es adoptar procedimientos para generar, desarrollar e implementar ideas nuevas 0 
comportamientos como respuesta a los cambios que surgen en el entorno. Yam et. al (2004) sostiene que es un conjunto de propiedades de las empresas, estas propiedades son capacidades y habilidades con las cuales pueden mejorar su gestión, desempeño y competitividad.

\section{Relación empírica entre la innovación y el desempeño organizacional}

Una investigación desarrollada en 12 empresas agroindustriales en Colombia (Toro et al., 2017), concluyó que las organizaciones implementan estrategias de innovación en sus procesos de actualización o renovación de equipos, después en productos y finalmente en la gestión de la empresa. Manifiestan que las organizaciones que implementan la innovación en sus procesos tienen un mejor desempeño al gestionar sus empresas, esto se refleja en la calidad, imagen, satisfacción del cliente y participación de mercado.

De igual forma, una investigación realizada en México, Chile, Colombia y Perú por García et al. (2016), evaluaron el efecto de la innovación en el crecimiento y rendimiento de las MiPyMes a través de cuestionarios aplicados a los directivos. Los resultados indican que el estado innovador impacta positivamente en el desempeño de las organizaciones. Las innovaciones se implementan en productos, procesos y gestión, lo que impacta en ventas, empleos y utilidades.

De acuerdo a la teoría e investigaciones previamente mencionadas, se propone la siguiente hipótesis:

H2: Las acciones de innovación que implementan las mipymes de Hidalgo, México, influyen en su nivel de desempeño organizacional.

\section{Valor al cliente}

Para poder conocer realmente a un cliente y poder retenerlo cuando es rentable, se necesita recabar información sobre el valor al cliente. Este concepto de gestión de relaciones en el área de mercadotecnia ha sido de gran importancia desde principios de los años 80. La retención de clientes es algo que debe preocuparle a las empresas si quieren desarrollar campañas de mercadotecnia enfocadas correctamente, por lo cual, no se deben tomar en cuenta sólo las predicciones del flujo de caja futuro, sino lo que el cliente realmente valora para poder continuar como consumidor de la empresa (Hwang et. al, 2004).

Distintos estudios realizados en los años 90 permiten identificar los inicios de la idea sobre el valor al cliente, tanto para comprender el comportamiento del consumidor como para el manejo de las estrategias de mercadotecnia (Kholi y Jaworsky, 1990; Narver y Slater, 1993; Zeithaml, 1988; Holbrook, 1999). Como anteriormente se mencionaba, las empresas empiezan a hacer conciencia de la importancia que tiene el valor al cliente porque esto comienza desde que una organización tiene orientación al mercado, con esto logran tener los conocimientos sufcientes para implementar y desarrollar estrategias de una manera eficaz y eficiente (Morgan y Strong, 1998).

Para Grant (1995), orientar una empresa hacia el valor al cliente se refiere al valor que es percibido por éste, de acuerdo a su experiencia con la organización, la cual es quien debe estimular esta experiencia. Valenzuela y Torres (2008) señalan que el valor al cliente se refiere a la capacidad de una organización para identificar y ordenar los elementos estratégicos que reciben de su mercado. Valenzuela y Castillo (2007) afirman que el valor del cliente para una empresa es el tiempo de relación que tiene un cliente con la organización y su facultad para atraer a nuevos clientes a través de sus referencias.

\section{Relación empírica entre el valor al cliente y el desempeño organizacional}

En la literatura revisada, se encontraron pocas investigaciones que relacionen directamente al constructo valor del cliente con la variable desempeño organizacional. Una de ellas es la realizada por Valenzuela y Villegas (2015), en la cual plantearon un modelo conformado por los constructos orientación al valor del cliente, cultura organizacional orientada al mercado, valor de marca y etica empresarial para entender cómo estos influyen en el desempeño organizacional. Los resultados arrojaron que existe una alta correlación entre las variables, y concluyen que es necesario que los ejecutivos y colaboradores trabajen para mejorar la satisfacción laboral del área 
de ventas y mercadotecnia, ya que a través de ésta es como se puede generar el valor de los clientes.

En una segunda investigación realizada por Gallarza et al., (2011) fue desarrollada en hoteles de España, se compararon dos modelos que relacionan el valor entregado, la orientación al cliente, la cultura de servicio y la percepción de la satisfacción del cliente. Concluyeron que la relación entre el valor y la satisfacción es inexistente a menos que incluya otras variables como la orientación al cliente y la cultura. La percepción de los clientes acerca del valor que reciben se encuentra relacionada con cómo los colaboradores entregan el servicio a los clientes de acuerdo a la orientación de la organización. Es por ello, que se puede formular la siguiente hipotesis:

H3: Las acciones sobre el valor al cliente que implementan las mipymes del estado de Hidalgo, México, se relacionan con su nivel de desempeño organizacional.

\section{METODOLOGÍA:}

La presente, es una investigación de carácter cuantitativo y correlacional, ya que pretende establecer la relación entre la innovación, la orientación al cliente, el valor al cliente y el desempeño organizacional de las empresas objeto de estudio. Así mismo, se trata de una investigación de diseño transversal, ya que la recolección de la información en las empresas se realizó en un momento determinado del tiempo (Hernández, Fernández y Baptista, 2010).

Por otra parte, en México, de acuerdo al número de empleados con el que cuenta las empresas se pueden clasificar como micro (0-10 personas), pequeñas (1150 personas), medianos (51-250 personas) y grandes (más de 250 personas) (INEGI, 2019). Para ese estudio se tomaron en cuenta establecimientos de diferentes puntos del estado de Hidalgo, México; sin importar su giro, únicamente que se consideraran mipymes (menos de 250 empleados). Se solicitó la participación de directores o propietarios de los establecimientos objeto de estudio. No se excluyó por género, edad, escolaridad u otra característica demográfica de los sujetos incluidos en la investigación.

Para la integración del cuestionario, se utilizaron distintas escalas validadas. Para medir la orientación al cliente, se tomaron ocho ítems propuestos por Deshpandé et al. (1993), para el constructo de innovación se utilizaron cinco ítems propuestos por Hyrley y Hult (1998), para medir el valor al cliente se utilizaron cuatro ítems propuestos por Narver y Slater (1990), Reinartz et al. (2004) y Valenzuela y Torres (2008). Finalmente, para medir el desempeño organizacional se utilizaron 10 ítems propuestos por Slavković y Babić (2013). Cada ítem utilizado en el cuestionario se midió a través de la escala Likert de cinco puntos, donde 1 representa totalmente en desacuerdo y 5 totalmente de acuerdo.

Este cuestionario se suministró a los sujetos de estudio, a través de la aplicación google forms. El periodo de recolección de información se llevó a cabo entre los meses de febrero a mayo de 2020, obteniéndose información de 32 unidades económicas del Estado de Hidalgo, México. La información recabada fue procesada utilizando el software estadístico Stata (Stata Corp) en su versión 15.

\section{RESULTADOS:}

El análisis de los resultados obtenidos mediante estadística descriptiva permite obtener una perspectiva general sobre el estudio y las empresas encuestadas. De acuerdo con ello, la mayoría de las unidades encuestadas (el 84\%) se consideran micro empresas, el $10 \%$ son pequeñas y el $6 . \%$ medianas. Respecto al giro del negocio, el $50 \%$, pertenece al sector servicios, el $47 \%$, pertenece al sector comercial y el $3 \%$ pertenece al sector industrial. Finalmente, la mayoría de las empresas (56\%) tiene más de 5 años de antigüedad, el $34 \%$ tiene una antigüedad de entre 1 y 5 años, y sólo el $10 \%$ tiene menos de un año de haber iniciado en el mercado. 
Ahora bien, para la validación del instrumento, es decir la medición de la consistencia entre los ítems, se hicieron las pruebas de alfa de Cronbach y de Fiabilidad compuesta. Siguiendo los criterios de George y Mallery (2003), quienes consideran que si el alfa de Cronbach es mayor a .8 es bueno; si es mayor a 0.7 es aceptable. Por su parte, la Fiabilidad Compuesta se considera adecuada cuando el valor de cada factor es mayor a 0.70 (Hair, et al., 2009).

Como se puede observar en la tabla 1, los niveles del alfa de Cronbach como de la fiabilidad compuesta, se encuentran por arriba de los niveles recomendados, a excepción del constructo valor al cliente, el cual se encuentra en los umbrales del nivel de aceptación, por lo cual, se decidió que continuará formando parte para el análisis de correlación.

Tabla 1. Medidas de confiabilidad de constructos

\begin{tabular}{lcc}
\hline Constructo & $\begin{array}{c}\text { Alfa de } \\
\text { Cronbach }\end{array}$ & $\begin{array}{c}\text { Fiabilidad } \\
\text { compuesta }\end{array}$ \\
\hline Orientación al cliente & 0.77 & 0.76 \\
Capacidad de Innovación & 0.77 & 0.84 \\
Valor al cliente & 0.68 & 0.68 \\
Desempeño Organizacional & 0.85 & 0.85 \\
\hline
\end{tabular}

Fuente: Elaboración propia, en base a resultados del software Stata

Por otra parte, se evaluaron las cargas factoriales de cada uno de los constructos, estas cargas indican el grado de correspondencia entre cada variable y el factor, y las cargas factoriales de 0.50 o mayores se consideran significativas (Hair, 2009). En ese sentido, se tuvo que prescindir de los ítems 1,5 y 6 del constructo orientación al cliente, el ítem 1 relacionado con la innovación; el ítem 1 de valor al cliente, así como los ítems 1,8 y 9 correspondientes al constructo desempeño organizacional (ver tabla 2).
Tabla 2. Estadísticas descriptivas y las cargas factoriales

\begin{tabular}{|c|c|c|c|}
\hline Variable & Media & $\begin{array}{l}\text { Desviación } \\
\text { estándar }\end{array}$ & $\begin{array}{c}\text { Cargas } \\
\text { factoriales }\end{array}$ \\
\hline \multicolumn{4}{|c|}{ Orientación al cliente } \\
\hline $\mathrm{OC2}$ & 4.41 & 0.67 & 0.56 \\
\hline OC3 & 4.38 & 0.83 & 0.51 \\
\hline OC4 & 4.19 & 0.64 & 0.50 \\
\hline OC7 & 4.25 & 0.76 & 0.85 \\
\hline OC8 & 4.69 & 0.59 & 0.65 \\
\hline \multicolumn{4}{|c|}{ Innovación } \\
\hline $\mathrm{Cl} 2$ & 4.34 & 0.75 & 0.75 \\
\hline $\mathrm{Cl} 3$ & 3.88 & 1.04 & 0.84 \\
\hline $\mathrm{Cl} 4$ & 4.16 & 0.88 & 0.62 \\
\hline $\mathrm{Cl} 5$ & 4.22 & 0.91 & 0.77 \\
\hline \multicolumn{4}{|c|}{ Valor al cliente } \\
\hline VC2 & 3.90 & 0.86 & 0.55 \\
\hline VC3 & 4.13 & 0.83 & 0.77 \\
\hline VC4 & 3.97 & 0.90 & 0.60 \\
\hline \multicolumn{4}{|c|}{ Desempeño organizacional } \\
\hline DO2 & 3.97 & 1.03 & 0.50 \\
\hline DO3 & 4.06 & 0.91 & 0.70 \\
\hline DO4 & 3.97 & 0.82 & 0.59 \\
\hline D05 & 4.25 & 0.80 & 0.94 \\
\hline D06 & 4.00 & 0.80 & 0.51 \\
\hline D07 & 4.34 & 0.75 & 0.61 \\
\hline D010 & 4.38 & 0.66 & 0.74 \\
\hline
\end{tabular}


Se llevó a cabo la correlación de Pearson entre los constructos orientación al cliente, innovación, valor al cliente y desempeño organizacional para probar las hipótesis planteadas. Como se observa en la tabla 3, se encontró una relación positiva y significativa entre la orientación al cliente con el desempeño organizacional, por lo cual $\mathrm{H} 1$ fue respaldada resultó ser la relación más fuerte entre los constructos mencionados. Por su parte la relación entre la innovación y el desempeño resultó no significativa, por lo cual $\mathrm{H} 2$ fue rechazada.

Finalmente, la relación entre el valor al cliente con el desempeño resultó ser positiva y significativa, con lo cual $\mathrm{H} 3$ no se rechaza. También puede observarse que el constructo denominado valor al cliente resultó tener una relación positiva y significativa con los demás constructos estudiados, mientras que la variable innovación no tuvo una relación significativa con la orientación al cliente, ni con el desempeño organizacional.

Tabla 3 Correlación entre constructos

\begin{tabular}{|c|c|c|c|c|}
\hline Constructos & 1 & 2 & 3 & 4 \\
\hline 1. Orientación al cliente & 1 & & & \\
\hline 2. Innovación & 0.27 & 1 & & \\
\hline 3. Valor al cliente & $0.67^{* * *}$ & $0.47^{*}$ & 1 & \\
\hline $\begin{array}{l}\text { 4. Desempeño } \\
\text { organizacional }\end{array}$ & $0.89 * * *$ & 0.32 & $0.62^{* * *}$ & 1 \\
\hline
\end{tabular}

Fuente: elaboración propia en base a resultados obtenidos en software Stata. ${ }^{* * *} p<.001 ;{ }^{* *} p<.01 ;{ }^{*} p<.05$

\section{DISCUSIÓN DE RESULTADOS:}

El objetivo de la presente investigación fue validar la relación entre la orientación al cliente, la innovación, el valor al cliente y el desempeño organizacional de las mipymes establecidas en el estado de Hidalgo, México. Para tal efecto, un cuestionario fue aplicado a 32 gerentes o dueños de unidades económicas de distintos giros. La fiabilidad de los resultados del instrumento se realizó a través de las pruebas de Alfa de Cronbach y Fiabilidad Compuesta.

De acuerdo a los resultados, se encontró que el constructo orientación al cliente tiene una relación positiva y significativa con el desempeño organizacional de las empresas encuestadas, con lo cual la hipótesis 1 fue respaldada. Este resultado se relaciona a lo reportado por Valenzuela y Martínez (2015), así como la investigación realizada por Rodríguez, et al (2015). Por su parte la hipótesis 2, tuvo que rechazarse, ya que sus resultados no fueron significativos. Este resultado recuerda a los hallazgos alcanzados por Singh et al., (2016), quienes observaron estos mismos resultados en empresas de Jordania. Por otra parte, este resultado entra en conflicto con las conclusiones obtenidas por Toro, et al (2017) en su investigación realizada en empresas Colombianas.

Finalmente, esta investigación reporta evidencia que respalda una relación positiva y significativa entre el constructo valor al cliente y el desempeño de las empresas estudiadas, con lo cual no se rechaza la hipótesis 3 . Este resultado se ajusta a las conclusiones obtenidas por Gallarza et al., (2011) que estudiaron esta relación en empresas hoteleras españolas.

Con estos resultados, se contribuye a mostrar evidencia empírica de la relación que existe entre la orientación al cliente, la innovación, el valor al cliente y el desempeño organizacional de micro, pequeñas y medianas empresas, en el contexto de países en desarrollo como es el caso de México.

Como parte de las limitaciones de esta investigación, se debe considerar que el estudio fue realizado mediante la respuesta obtenida de 32 unidades económicas asentadas en el estado de Hidalgo, México, lo anterior debido a las restricciones a la movilidad de las personas y el cierre temporal de 
diversos negocios, derivada de la pandemia ocasionada por el virus Covid-19 que ha afectado a las actividades cotidianas a nivel global.

\section{REFERENCIAS:}

Acevedo, D., Hirane, I., \& Morovic, J. (2007). El Impacto de la Orientación al Cliente en la Cultura y el Desempeño de las Empresas de Servicio. Un Enfoque Cualitativo. Universidad de Chile, 97-98. https://doi.org/10.5354/anuc.v0i97-98.11040

Afcha, S. (2012). Innovaciones organizacionales y su efecto sobre el desempeño empresarial. Revista Venezolana de Gerencia, 16(56). https://doi.org/10.31876/revista.v16i56.10649

Almanza, R., Calderón, P., Vargas, J. G., Casas, R., \& Palomares, F. (2016). Aprendizaje y Desempeño Organizacional bajo el enfoque de las Teorías Organizacionales. 13(1), 83-94.

Arshad, A. M., Wang, J., \& Su, Q. (2016). Investigating The Mediating Role Of Service Innovation In Firm Performance: An Empirical Research. 32(2), 461-479.

Churchill Jr, G. A., Ford, N. M., Hartley, S. W., \& Walker Jr, O. C. (1985). The determinants of salesperson performance: A metaanalysis. Journal of marketing research, 22(2), 103-118.

Damanpour, F. (1989). The relationship between types of innovation and organizational performance. Journal of Management Studies, 26 (6), 587-01.

Damanpour, F. (1996). Organizational complexity and innovation: developing and testing multiple contingency models. Management Science, 42 (5): 693-16.

Deshpandé, J., Farley, U. y Webster, J. (1993), Corporate Culture, Customer Orientation, and Innovativeness in Japanese Firms: A Quadrad Analysis. Journal of Marketing, (57), 22-27.
Gallarza, M., Gil, I., \& Arteaga, F. (2011). El valor entregado, la cultura de servicio y la orientación al cliente en los hoteles de España. Papers de Turisme, 0(49), 7-24.

García, D., Gálvez, E., \& Maldonado, G. (2016). Efecto de la innovación en el crecimiento y el desempeño de las Mipymes de la Alianza del Pacífico. Un estudio empírico. Estudios Gerenciales, 32(141), 326-335. https://doi.org/10.1016/j.estger.2016.07.003

George, D., \& Mallery, P. (2003). SPSS for Windows step by step: answers to selected exercises. A simple guide and reference, 63, 1461-1470.

González, Ó., \& González, J. (2004). Orientación Al Mercado Cultural vs Operativa y Desempeño Empresarial Objetivo vs Subjetivo: Perspectiva de Producción. Universidad de Salamanca, 129.

Grant, R. (1995). Contemporarystrategy analysis: concepts, techniques, applications. Cambridge.

Gutiérrez, M. del C., Sapién, A. L., \& Piñón, L. C. (2013). Desempeño Organizacional De Microempresas En México. European Scientific Journal, $\quad 9(28)$, 102-112. https://doi.org/10.19044/esj.2013.v9n28p\%p

Hair, J. F., Black, W. C., Babin, B. J., y Anderson, R. E. (2009). Multivariate Data Analysis 7 ma edición. Nueva York: Pearson Prentice Hall

Hernández, A. (2012). El Valor Percibido por El Consumidor: Conceptualización $Y$ Variables Relacionadas. Camino para El Éxito de un Negocio. 3C Empresas, 2-7.

Hernández, R., Fernández, C., y Baptista, M. (2010). Metodología de la investigación (5ta ed.). 75 México: Mc Graw-Hill.

Holbrook, M.B. (1999). Consumer value. A framework for analysis and research. Routledge, Londres. 
Reinartz, W., Krafft, M. y Hoyer, W. (2004). The customer relationship management process: its measure-ment and impact on performance. Journal of Marketing Research. 41(3), 293-305.

Rodríguez, P., Ramos, A., \& Pastor, M. del P. (2015). La orientación al mercado de las mipymes de San Luis Potosí. Revista Electrónica Nova Scientia, 7, 436-461.

Seaden, G., et ál. (2003). Strategic decisions and innovation in construction. Construction Management and Economics, 21, (6): 603-12.

INEGI. (2019). Censos Económicos 2019. https://www.inegi.org.mx/contenidos/programas/ ce/2019/doc/pro_ce2019.pdf

Kohli, A. y Jaworoski, B. (1990). Market Orientación: The Construct, research propositions and managerial implications. Journal of Marketing. 53-70

Llonch, J. (1993). Orientación al Mercado y Competitividad de la Empresa (No. 658.5 L792). Eada Gestión.

Machorro, F., Mercado, P., Cernas, D. A., \& Romero, M. V. (2015). Influencia del capital relacional en el desempeño organizacional de las instituciones de educación superior tecnológica. Revista Innovar Journal, 26(60), 35-50. https://doi.org/10.15446/innovar.v26n60.55531. L20

Morgan, R. E. y Strong, C.A. (1998). Market orientation and dimensions of strategic orientation. European Journal of Marketing, 32, 1051-1073.

Narver, J.C. y Slater S. (1990). The effect of a market orientation on business profitability. Journal of Marketing. 22-35.

Nwankwo, S. (1995). Developing a customer orientation. Journal of consumer marketing.

Ogunyomi, P., \& Bruning, N. S. (2015). Human resource management and organizational performance of small and medium enterprises (SMEs) in Nigeria. International Journal of Human Resource Management, 27(6), 612-634. https://doi.org/10.1080/09585192.2015.1033640

Porter, M. (1991). Ventaja Competitiva Creación y Sostenimiento de un Desempeño Superior (T. F. PRESS (ed.)). Rei Argentina, S.A.
Slavković, M., y Babić, V. (2013). Knowledge management, innovativeness, and organizational performance: Evidence from Serbia. Economic Annals, 58(199), 85-108. https://doi.org/10.2298/EKA1399085S

Singh, S., Darwish, T. K., \& Potocnik, K. (2016). Measuring Organisational Performance A Case for Subjective Measures. British Journal of Management, 27(1), 214-224. https://doi.org/10.1111/14678551.12126/abstract

Toro, N., Castaño, J. M., \& López, Y. (2017). Estado innovador de empresas medianas y pequeñas agroindustriales de Manizales y su relación con el desempeño organizacional. Ánfora, 24(42), 67-93. https://doi.org/10.30854/anf.v24.n42.2017.164

Tushman, M., \& Nadler, D. (1986). Organizing for innovation. California review, 28(3), 74-92. management

Valenzuela, L. y Castillo, E. (2007). El cambio del marketing en dirección a una nueva era de gestión

Valenzuela, L. y Martínez, C. (2015). Orientación al Cliente, Tecnologías de Información y Desempeño Organizacional: Caso empresa de consumo masivo en Chile*. Revista Venezolana de Gerencia, 20(70). https://doi.org/10.31876/revista.v20i70.19999

Valenzuela, L. y Torres, E. (2008). Gestión empresarial orientada al valor del cliente como fuente de ventaja competitiva. Propuesta de un modelo explicativo. Estudios Gerenciales, 24(109), 65$86 . \quad$ https://doi.org/10.1016/s01235923(08)70053-6 
RELACIÓN DE LA ORIENTACIÓN AL CLIENTE, DE VALOR AL CLIENTE Y LA INNOVACIÓN SOBRE EL DESEMPEÑO ORGANIZACIONAL. UN ESTUDIO EN MIPYMES DEL ESTADO DE HIDALGO, MÉXICO

Valenzuela, L. y Villegas, F. (2015). Orientación al Valor del Cliente y el Desempeño

Organizacional: Propuesta de un Modelo Explicativo. Administración \& Finanzas, 6(7).

Yam, R. C. M., Guan, J. C., Pun, K. F., and Tang, E. P. $Y$. (2004). An audit of technological in- novation capabilities in Chinese firms: some empirical findings in Beijing, China. Research Policy, 33 (8), 1123-1140.

Yamakawa, P. y Ostos, J. (2011). Relación entre innovación organizacional y desempeño organizacional. Universidad \& Empresa, 13(21), 93-115.

http://www.redalyc.org/articulo.oa?id=18722242 0005

Ynzunza, C. y Izar, J. (2016). Estrategia de negocio, gestión de recursos humanos y desempeño organizacional. Investigación y Ciencia: De La Universidad Autónoma de Aguascalientes, 69, 47-53.

Ynzunza, C. y lzar, J. (2013). Estrategia, Orientación al Mercado y Desempeño Organizacional. Conciencia Tecnológica, 45, 5-11.

Zeithaml, V. (1988). Consumer perceptions of price, quality, and value: a means-end model and synthesis of evidence. Journal of Marketing, 52, pgs. 2-22. 